\title{
Inline Inspection of Ceramic Tape Casting Processes by Means of Optical, Eddy Current and Machine Learning Methods
}

\author{
Manuela Heymann ${ }^{1}$, Stefan Münch ${ }^{1}$, Martin Schulze ${ }^{1}$, Beate Capraro², Dirk Schabbel2, Vidit Gupta ${ }^{1}$, \\ Andy Vogel2, Christiane Schuster ${ }^{1}$, Thomas Härtling ${ }^{1,3}$ \\ ${ }^{1}$ Fraunhofer Institute for Ceramic Technologies and Systems IKTS, Maria-Reiche-Strasse 2, 01109 \\ Dresden, Germany, \\ 2 Fraunhofer Institute for Ceramic Technologies and Systems IKTS, Michael-Faraday-Strasse 1, \\ 07629 Hermsdorf, Germany \\ ${ }^{3}$ Technische Universität Dresden, Institute of Solid State Electronics, Mommsenstraße 15, 01069 \\ Dresden, Germany \\ thomas.haertling@ikts.fraunhofer.de
}

\begin{abstract}
Summary:
Tape casting is a ceramic forming technology used to produce planar ceramic components by means of a doctor-blade process. However, industrial casting plants are currently not equipped with inline measurement tools which allow detecting of defects and determine quality parameters of the ceramic tape. Here we use laser triangulation, camera-based monitoring, and eddy current measurement tools to acquire different tape quality parameters. Results of the implementation of these tools and the insights gained by applying machine learning algorithms for automated defect detection and classification are presented.
\end{abstract}

Keywords: tape casting, process monitoring, ceramics, optical inspection, eddy current, machine learning

\section{Introduction}

Tape casting is a high-throughput method for producing large, flexible tapes of functional materials very efficiently and cost-effectively in roll-to-roll processes. Applications of such tapes range from classic ceramic microsystem technologies (LTCC and HTCC) and the current strategic field of battery research to filtration, gas separation, and a variety of special functionally tapes. Finely ground ceramic powders are dispersed under addition of suitable dispersants, organic binders, and plasticizers. The resulting viscous casting slurry undergoes a doctor-blade process and subsequent drying done in a drying channel of several meters of length. The result is a very thin ceramic tape with flat surface.

The tapes produced can show several defects such as air pockets, bubble formation, large particle / object inclusions, density fluctuations of the slurry and fluctuations of the tape thickness. At present, industrial casting plants are not equipped with process monitoring tools which allow detecting defects and determine quality parameters inline. As a result, defects and parameters outside specification margins are only detected after the manufacturing process and the operators face high costs due to returned batches.

The authors intend to evaluate measuring methods for defect detection, to develop an inline application and in future adapt the information of the different methods to an overall description of the tape quality.

The inspection methods used here are laser triangulation for thickness determination at the beginning and end of the drying channel (measurement of wet and dry film thickness), camera-based optical inspection, and eddy current measurements at the end of the drying line (detection of inclusions, material defects, holes, deviation in dielectric constant). The methods have been evaluated and optimized regarding hardware, location and method of implementation, data generation and evaluation, and were integrated in a demonstration casting plant.

In this contribution we show results of the different monitoring systems and first results on 
defect prediction based on machine learning algorithms and the effects on operating tape casting machines.

\section{Measurement System}

For thickness measurement, four laser triangulation sensors (Keyence LK-H087) were installed (a static reference and a dynamic sensor each at the beginning and the end of the drying line). Hence, they were applied in differential mode to determine the film height. Furthermore, optical inspection was carried out with a single line camera (Teledyne Dalsa Linea) and alternating illumination in reflection and transmission mode. Images were acquired exploiting the movement of the ceramic foil under the line sensor of the camera. This system was intended to detect defects in the ceramic foil. As a third component, an inline eddy current array probe setup by Fraunhofer IKTS [1] was installed at the backend of the drying line. This system allows to evaluate especially the (di)electric properties of the cast foil. It was operated in reflection mode.

\section{Results}

After the implementation of the described measurement tools results were obtained which allow a first inline characterization of the ceramic tape. For demonstration, a sample casting with an $\mathrm{Al}_{2} \mathrm{O}_{3}$ slurry was carried out. The tape width was $150 \mathrm{~mm}$, the doctor-blade gap $400 \mu \mathrm{m}$ (tape wet thickness) and casting velocity $0.4 \mathrm{~m} / \mathrm{min}$.

The thickness measurement was proven feasible at the backend of drying line (see results depicted in Fig. 1).

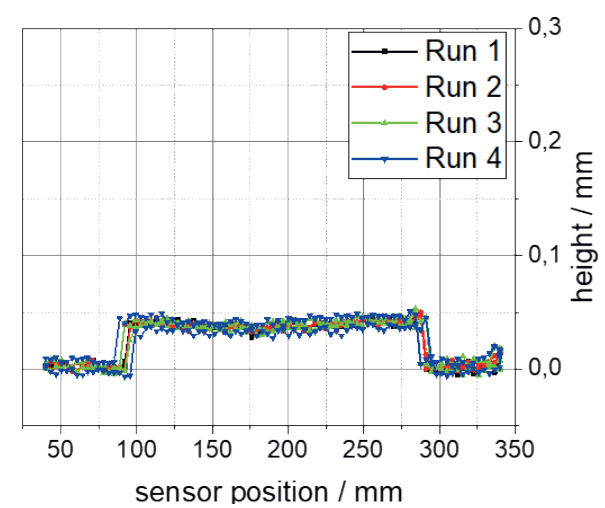

Fig. 1 Feasibility proof of inline thickness measurement of the dry ceramic green tape.

The camera-based optical inspection tool allows to detect specific defects. As an example, a small defect (a hole) is shown in Fig. 2 in reflection mode (left) and transmission mode (right). These images were used as training database for image classification with machine learning (ML) algorithms. The following steps have been pursued: image classification and labeling, image preprocessing, setting up of ML model including training and evaluation, and assessment of accuracy. For binary classification (either no defect or any kind of defect), a model based on the EfficientNet B5 showed best results. For multiclassification (four defect classes), best results were achieved by applying a U-Net model which uses an encoder and decoder approach.

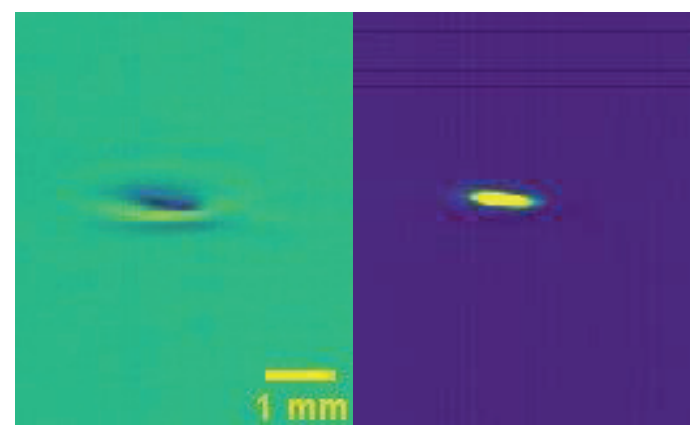

Fig. 2 Optical inspection with alternating illumination. Left: reflecting mode. Right: transmission mode. In both images a small defect (hole) is visible which leads to rejection in further material processing.

Finally, a first test of the eddy current setup allows to detect NdFeB particles in the foil. They are marked as blue spots in the image in Fig. 3.

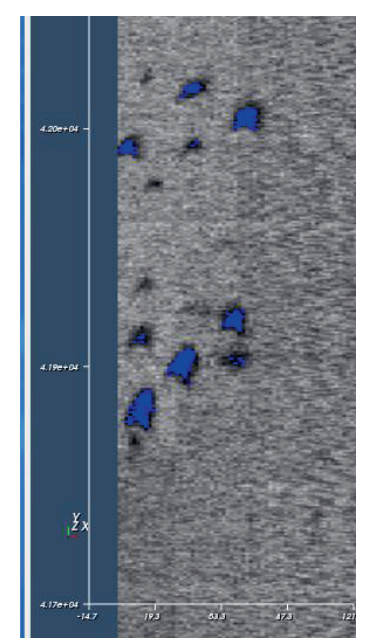

Fig. 3 Detection of $\mathrm{NdFeB}$ particles (blue spots) by means of the eddy current system.

Based on the current results and the ongoing work, our contribution will provide a detailed description of the measurement system in use, a classification of detectable defects, results on defect detection based on machine learning algorithms, and a discussion of the benefits by fusing the data of all three measurement tools.

\section{References}

[1] M. Wild, A. du Barét, S. Reuber, M. Schulze, H. Heuer, M. Wolter, A. Michaelis, Analysis and 
monitoring of the percolating network of battery electrode films during the drying process using eddy current technology, Lecture, International Battery Production Conference IBPC 2019,

5.11.2019 